\title{
Non-steroidal anti-inflammatory drugs for the treatment of pain and immobility-associated osteoarthritis: consensus guidance for primary care
}

Ade Adebajo

\begin{abstract}
Background: Osteoarthritis is a common presentation in primary care, and non-selective non-steroidal antiinflammatory drugs (sometimes also referred to as traditional NSAIDs or tNSAIDs) and selective cyclo-oxygenase 2 inhibitors (COX-2 inhibitors) are commonly used to treat it. The UK's National Institute for Health and Clinical Excellence (NICE) recommends taking patient risk factors into account when selecting a tNSAID or a COX-2 inhibitor, but GPs have lacked practical guidance on assessing patient risk.
\end{abstract}

Methods: A multi-disciplinary group that included primary care professionals (PCPs) developed an evidence-based consensus statement with an accompanying flowchart that aimed at providing concise and specific guidance on NSAID use in osteoarthritis treatment. An open invitation to meet and discuss the issue was made to relevant healthcare professionals in South Yorkshire. A round table meeting was held that used a modified nominal group technique, aimed at generating opinions and ideas from all stakeholders in the consensus process. A draft developed from this meeting went through successive revisions until a consensus was achieved.

Results: Four statements on the use of tNSAIDs and COX-2 inhibitors (and an attached category of evidence) were agreed: 1) tNSAIDs are effective drugs in relieving pain and immobility associated with osteoarthritis. COX-2 inhibitors are equally effective; 2) tNSAIDs and COX-2 inhibitors vary in their potential gastrointestinal, liver, and cardio-renal toxicity. This risk varies between individual treatments within both groups and is increased with dose and duration of treatment; 3) COX-2 inhibitors are associated with a significantly lower gastrointestinal toxicity compared to tNSAIDs. Co-prescribing of aspirin reduces this advantage; 4) PPIs should always be considered with a tNSAID and with a COX-2 inhibitor in higher Gl risk patients. An accompanying flowchart to guide management was also agreed.

Conclusions: Individual patient risk is an important factor in choice of treatment for patients with osteoarthritis and the consensus statement developed offers practical guidance for GPs and others in primary care. Where there are clinical uncertainties, guidance developed and agreed by local clinicians has a role to play in improving patient management.

\section{Background}

Osteoarthritis is a common presentation in primary care, responsible for an estimated $2.4 \%$ of all GP consultations in the UK, and a major contributor to the annual 10.1 million consultations for musculoskeletal conditions overall [1]. Those with osteoarthritis have an increased risk of death from any cause, and particular for mortality related to cardiovascular disease and dementia [2].

\footnotetext{
Correspondence: A.O.Adebajo@sheffield.ac.uk

Academic Rheumatology Group, Faculty of Medicine, University of Sheffield and Barnsley Hospital NHS Foundation Trust, Gawber Road, Barnsley S75 2EP
} UK

(C) 2012 Adebajo; licensee BioMed Central Ltd. This is an Open Access article distributed under the terms of the Creative Commons Attribution License (http://creativecommons.org/licenses/by/2.0), which permits unrestricted use, distribution, and reproduction in any medium, provided the original work is properly cited. (tNSAIDs) are effective drugs in relieving pain and inflammation associated with osteoarthritis and other musculoskeletal conditions, and in promoting mobility and physical activity. They are commonly prescribed in primary care. Agents that selectively inhibit cyclo-oxygenase 2 (COX-2 inhibitors) are equally effective [3-6].

In its guidance on osteoarthritis the National Institute for Health and Clinical Excellence (NICE) recommends initial management with education, advice and information, strength and aerobic exercise, and weight loss for overweight and obese patients, followed by treatment 
with paracetamol or topical NSAIDs if initial treatment is not successful [7].

Where paracetamol or topical NSAIDs are ineffective for pain relief, NICE suggests consideration of an oral non-selective NSAID or a COX-2 inhibitor, prescribed with a proton pump inhibitor (PPI). The NICE guidance suggests taking individual patient risk factors including age into account when selecting a tNSAID or COX-2 inhibitor, with assessment and ongoing monitoring of risk factors.

While the effectiveness of both tNSAIDs and COX-2 inhibitors is similar, the potential adverse effects vary. In particular COX 2 inhibitors are associated with a lower risk of gastrointestinal adverse effects compared to tNSAIDS, and there is some evidence that naproxen is associated with a lower cardiovascular risk than other tNSAIDs $[6,8]$.

The NICE guidance is a useful basis for clinical practice, but in their communications with GPs, for example in referral letters and at educational events, rheumatologists in South Yorkshire identified some uncertainty about its detailed application in the wake of rapidly-evolving new evidence on the risks and benefits of tNSAIDs and COX-2 inhibitors. In particular GPs were unsure about how to assess the risk status of patients who could benefit from a tNSAID or COX-2 inhibitor, and so to identify the most appropriate treatment. Following the high-profile withdrawal of the COX-2 inhibitor rofecoxib in 2004 in the wake of concerns about cardiovascular safety [9], and the subsequent withdrawals of valdecoxib (because of a high rate of serious skin adverse effects and concerns about cardiovascular safety) [10] and lumiracoxib (because of severe hepatic adverse events) [11] some GPs believed that all COX-2 inhibitors had been withdrawn.

To address these uncertainties and in the light of additional clinical evidence, we therefore developed an evidence-based consensus statement, and an accompanying management flowchart to provide more specific guidance for GPs and others working with osteoarthritis patients in primary care. The aim of the consensus process was to develop a practical, evidence-based statement, in line with existing NICE guidance that would help GPs to identify the risk status of patients with osteoarthritis and, where appropriate, to provide the most effective appropriate tNSAID or COX-2 treatment for them.

\section{Methods}

The lead physician for the consensus statement (AOA), issued an open invitation by email to relevant local specialists and primary care physicians. In response to this invitation a group comprising a rheumatologist, a consultant cardiologist, a consultant gastroenterologist, a hospital pharmacist and three primary care physicians with an interest in pain and/or rheumatology attended a round table chaired by the lead physician (a consultant rheumatologist). The key requirement for the project was that the major specialties related to this topic were represented. The meeting used a modified nominal group technique in order to generate opinions and ideas from all the relevant stakeholders who had expertise in primary care, rheumatology, cardiology, gastroenterology, pharmacy and pain relief.

Nominal group technique is a decision making method that typically involves an initial silent generation of ideas by individuals, without discussion or consultation with others, followed by an uncritical sharing of ideas, group discussion and then a ranking or evaluation of ideas [12]. It is regarded as an effective method of dealing with a relatively closed issue, as here.

After the meeting the lead physician developed a draft consensus statement and algorithm that went through several successive drafts until a consensus statement and accompanying flowchart was agreed by at least $90 \%$ of the participants. In practice disagreements were minor and the consensus statement was agreed by all participants.

The consensus group rated its recommendations (A strongest, D weakest) based on the quality of the supporting evidence (with the strongest being evidence from a meta-analysis of randomised controlled trials, and the weakest being expert opinion).

The consensus statement and the flowchart has been circulated to GPs and used in training activities.

\section{Results}

The consensus statement emphasised that tNSAIDs and COX-2 inhibitors are effective at treating symptoms of pain and immobility associated with osteoarthritis, but that they vary in their potential for adverse effects, particularly GI, cardiovascular, hepatic and renal. This risk varies between individual treatments and increases with dose and duration of treatment [6].

\section{Recommendations}

Four statements were developed for application in primary care, three supported by category A evidence and one by category $\mathrm{C}$ evidence (see Table 1 ).

We also developed a flow chart illustrating a clinical pathway for patients with osteoarthritis, based on NICE guidance, and the additional work of the South Yorkshire Clinical Consensus group (see Figure 1).

\section{Risk prediction}

The flowchart recommends calculating cardiovascular risk prediction according to the Joint British Societies guidelines on the prevention of cardiovascular disease in clinical practice (JBS 2), based on calculations derived from the Framingham study [13], and familiar to GPs in 
Table 1 Use of non-steroidal anti-inflammatory drugs

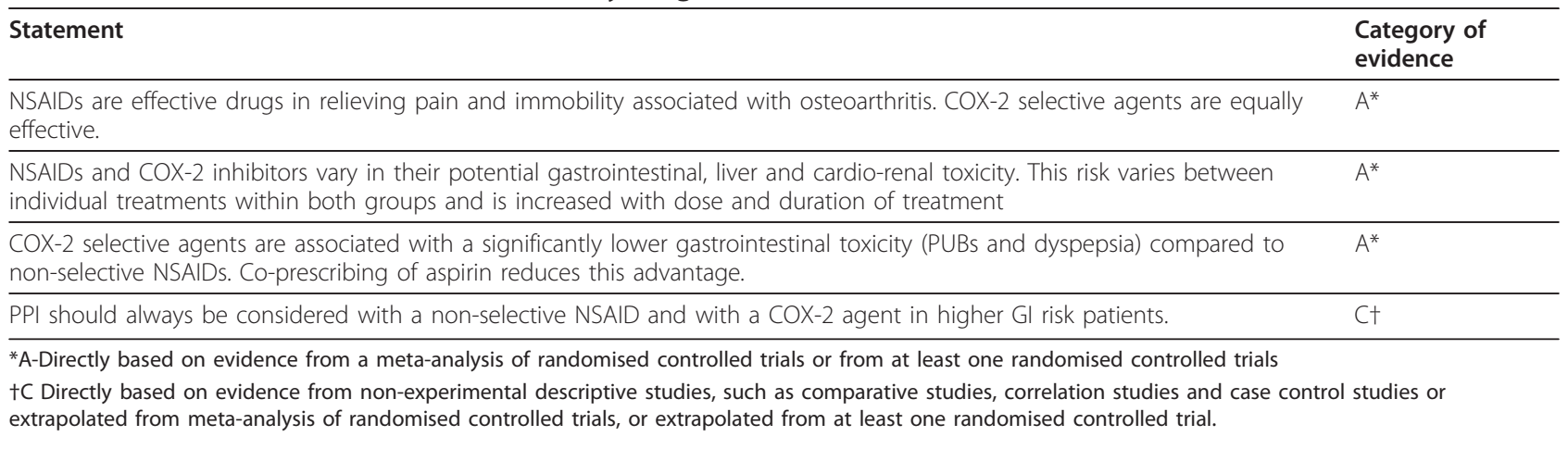

other contexts, such as decisions about statin prescribing [14]. They are based on an algorithm that uses a patient's age, systolic blood pressure, total cholesterol to HDL cholesterol ratio, and smoking status to calculate a 10 year risk of cardiovascular disease.

The NHS Clinical Knowledge Service has identified the following patient groups at increased risk for gastrointestinal adverse effects from oral non-selective NSAIDs:

Older age: the risk doubles with every decade after the age of 55

Male sex: the risk of an upper GI complication is twice as high in men than women

History of GI disorder such as gastroduodenal ulcer, GI bleeding

Use of medications such as aspirin, warfarin, oral corticosteroids, selective serotonin reuptake inhibitors, venlafaxine or duloxetine

Serious comorbidity such as cardiovascular disease, hepatic or renal impairment, diabetes or hypertension

Prolonged NSAID use

Use of maximum dose NSAID

Presence of Helicobacter pylori infection

Excessive alcohol use

Heavy smoking[15].

The consensus group recommended this guidance as a means of identifying GI risk in patients with osteoarthritis.

The groups identified by the Clinical Knowledge Service are:

Naproxen $500 \mathrm{mg}$ bd or low dose ibuprofen $(<1,200$ $\mathrm{mg} /$ day) plus a proton pump inhibitor (PPI) are recommended as first choice NSAIDs where patients are at low GI risk and moderate CV risk [7,16,17]. Both ibuprofen and naproxen may inhibit the antiplatelet action of aspirin and so other agents may be preferred in patients already receiving low-dose aspirin for cardiovascular prophylaxis who are likely to be at higher CV risk [18].

\section{Recent evidence on opioid analgesics}

We identified concern about the potential risks of tNSAIDs and COX-2 inhibitors that resulted in some GPs substituting opioid analgesics for osteoarthritis, perhaps unaware of the significant risks associated with opioid use. In the light of new evidence, the consensus statement is cautious on the use of opioid analgesics, and recommends they be restricted to patients with serious or absolute contraindications to tNSAIDs and COX-2 inhibitors [19-21].

Recent research has questioned whether the initial acute efficacy of opioid analgesics is sustained when used for long-term treatment over weeks and months. In addition, since the publication of the NICE guidance in 2008 concern has been expressed about their riskbenefit ratio in long term treatment of chronic musculoskeletal pain. A recent review of more than 36,000 prescriptions found an significantly increased cumulative risk over 12 months of cardiovascular events (myocardial infarction, stroke, hospitalisation for heart failure, coronary vascularisation and out of hospital cardiac death) for patients taking opioid analgesics compared to non-selective NSAIDs $(p<0.001)$ and to COX-2 inhibitors $(p=0.004)$ [21]. There was, similarly an increased risk of fractures, admission to hospital for safety events, and all-cause mortality for those taking opioids compared to non-selective NSAIDs or COX-2 inhibitors. There was an increased risk of upper or lower GI bleeding for opioids compared to COX-2 inhibitors ( $p=$ 0.04 ). The number needed to harm reported in this study was small for opioids, and clinically relevant.

\section{Diclofenac}

In a departure from the NICE guidance, which does not differentiate explicitly between different tNSAIDs, the consensus statement explicitly recommends against the 


\section{Management of osteoarthritis flowchart}

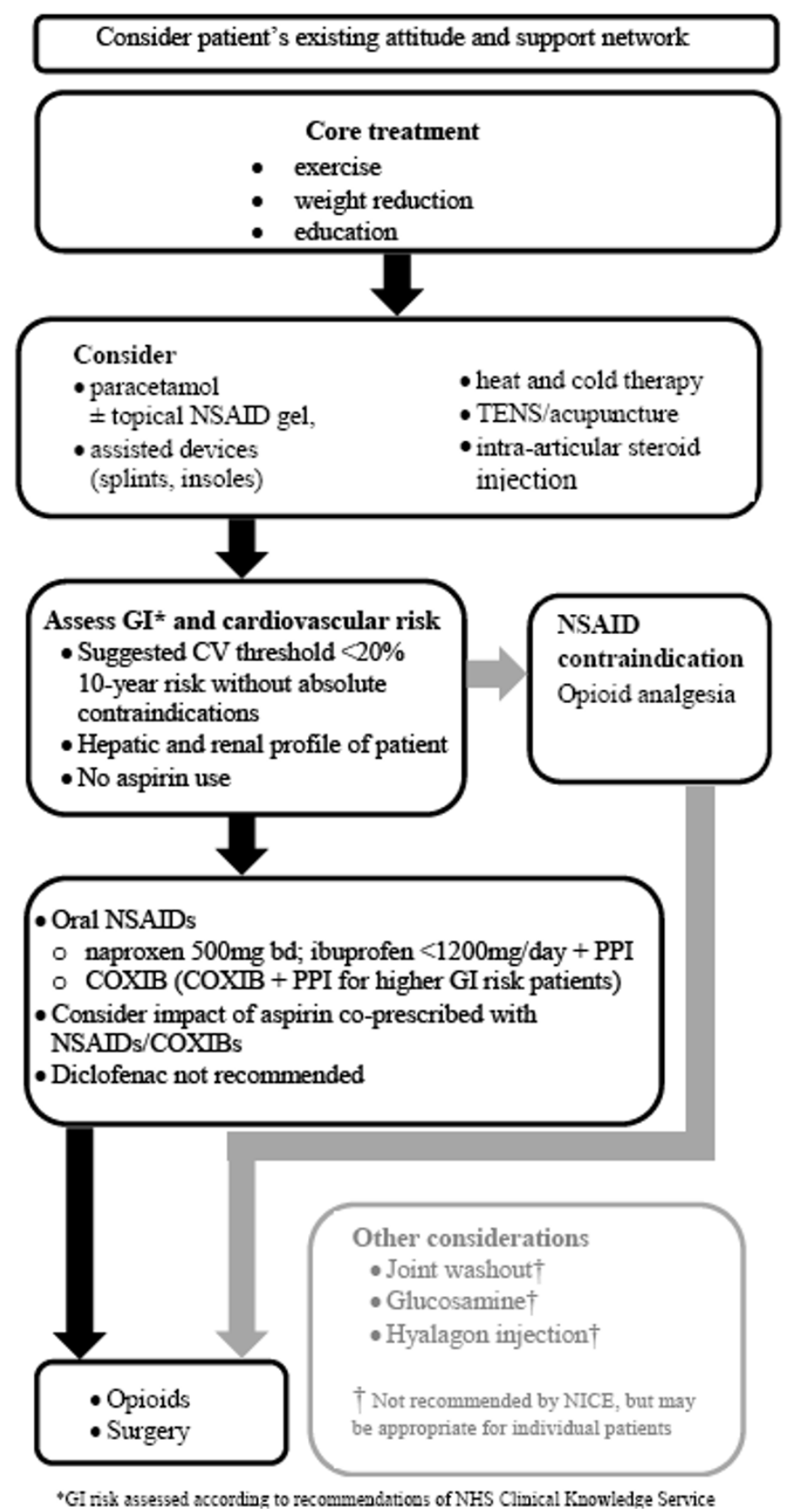

Figure 1 Management of osteoarthritis flowchart.

use of diclofenac. The decision for this additional recommendation was based on the strength of emerging evidence (largely published after the development of the NICE guidance) suggesting a higher cardiovascular risk such as stroke, cardiovascular death and myocardial infarction with diclofenac than other tNSAIDs and selective COX-2 inhibitors [8,22,23]. This emerging evidence suggests that it is prudent to take a precautionary 
approach and recommend the choice of one of the several alternative treatments to diclofenac when appropriate for new patients.

A retrospective population-based nested case-control analysis of data from the clinical records of more than 7 million patients registered with 468 UK general practices found a $55 \%$ increased risk of MI for those taking diclofenac, compared to those taking no tNSAIDs or COX-2 inhibitors in the previous 3 years $(p<0.05)$ [22]. The increased risk for ibuprofen was $24 \%$ and for the now withdrawn selective COX-2 inhibitor rofecoxib was $32 \%$ (both $p<0.05$ ) [22]. For diclofenac the number needed to harm over a year was 521 treated patients for every additional myocardial infarction, compared to 1,005 for ibuprofen and 695 for rofecoxib. An observational study found a 5.54-fold increase in the risk of death and a 2.24-fold increase in the risk of admission to hospital with myocardial infarction in heart failure patients taking $>100 \mathrm{mg}$ a day of diclofenac [23]. In a recent study of a population of patients who had already had a myocardial infarction, diclofenac was identified as the tNSAID with the highest risk of death or recurrent MI (HR3.26; 95\%CI2.57-3.86) - about twice the risk of treatment with any tNSAID (HR1.45;95\%CI1.29-1.62) [24].

\section{Selective COX-2 inhibitors}

COX-2 inhibitors were recommended for patients identified to be at risk from GI toxicity but not at significant CV risk ( $<20 \%$ 10-year risk of an event according to the Joint British Societies risk score [14]). There is evidence that both COX-2 inhibition and use of a non-selective NSAID plus PPI can reduce the risk of upper GI adverse events [25-27], and evidence from a large prospective randomised controlled trial of high risk patients that COX-2 inhibitors may prevent gastrointestinal adverse effects to a greater extent than a combination of tNSAID and PPI [28]. This RCT, of patients with osteoarthritis or rheumatoid arthritis who had a previous gastroduodenal ulcer and allocated to treatment with celecoxib or diclofenac and omeprazole, found a significant difference between the proportion of patients on celecoxib who developed a clinically significant upper or lower GI event (20/2238, 0.9\%), and those who developed an event on tNSAID plus PPI treatment (81/2246, 3.8\%), $p<0.0001$ [28].

\section{Future research}

One outcome of reviewing national guidelines in a local context as we did is coming across gaps in recommendations, often because of a lack of supporting clinical evidence. A number of clinical uncertainties were identified in developing the consensus statement, where future research may be warranted. These included:
- The efficacy, safety and cost effectiveness of COX2 inhibitors with and without PPI treatment versus naproxen or ibuprofen with and without PPI treatment

- The CV safety of COX-2 inhibitors versus tNSAIDs, including use of the $20 \%$ risk over 10 years threshold for CV appropriate NSAID prescribing.

$\circ$ The clinical effects of COX-1 inhibition and the pathogenesis of small bowel damage.

The first of these questions is addressed by the Prospective Randomized Evaluation of Celecoxib Integrated Safety vs. Ibuprofen or Naproxen (PRECISION). It is a large-scale trial expected to recruit 20,000 participants that should provide useful information about cardiovascular safety of non-selective NSAIDs and selective COX-2 inhibitors [29]. Results are scheduled for publication in 2014 [30].

\section{Discussion}

We aimed to produce clear, simple short guidance that was easy to use and developed in a way that meant that local clinicians would have a sense of local ownership. These characteristics have been identified by GPs as factors likely to make them refer to a guideline [31].

Clinical guidelines can help improve the quality of care when there is a lack of clarity about appropriate clinical practice and when scientific evidence can provide an answer [32]. The uncertainty about the use of non-selective, traditional NSAIDs and COX-2 inhibitors recognised by specialists in South Yorkshire makes the development of these guidelines an appropriate response to potential suboptimal management of patients with osteoarthritis. The consensus statement and accompanying flowchart make a number of practical and achievable recommendations to improve clinical treatment in primary care.

When choosing to manage osteoarthritis with tNSAIDS and COX-2 inhibitors, GPs should make an appropriate assessment including ongoing monitoring of GI and CV risk to maximise the benefits and minimise the risks of treatment. This consensus statement identifies practical ways for GPs to do that. Risk profile can be influenced by a number of factors including dose, concurrent aspirin use and age [33]. The lowest effective dose should be used for the shortest possible duration, as chronic treatment is associated with increased risk [34].

The reasons for the successful development of this local consensus statement include its restricted focus on a particular area of clinical uncertainty, its involvement of a wide range of stakeholders, and the delivery of a concise and practical clinical pathway. 


\section{Conclusions}

National clinical guidelines are effective ways to bring about improvements in the quality of care, but they do not always provide the practical guidance that primary care professionals require. Where there are uncertainties in clinical practice that might affect the quality of patient care, local guidance developed by local groups of clinicians has a role to play in health improvement.

Ascertaining individual patient risk is important when choosing treatment for patients with osteoarthritis, and the South Yorkshire consensus statement provides practical guidance for GPs and others in primary care to measure risk and guide therapeutic decisions.

\section{Acknowledgements \\ AA would like to acknowledge the members of the multidisciplinary group who contributed to the round table meeting including the following individuals: Dr Ollie Hart, GP, Sheffield; Dr Martin Johnson, GP/GPwSI pain, Barnsley; Dr lan Hall, Cardiologist, Northern General Hospital, Sheffield and Dr Robert Stevens, Rheumatologist, Doncaster. Others who took part felt that their contribution did not require individual acknowledgement. All members of the multidisciplinary group are aware of this publication, and have consented to its publication. \\ Mark Pownall of inScience Communications, a Wolters Kluwer business, provided medical writing assistance, which was funded by Pfizer.}

\section{Authors' contributions}

AA had involvement in all aspects and stages of the drafting, completion and approval of this manuscript.

\section{Competing interests}

AA has received unrestricted educational funding from various pharmaceutical companies including Pfizer. Funding for the medical writing of this manuscript was provided by Pfizer.

Received: 24 June 2011 Accepted: 20 March 2012

Published: 20 March 2012

\section{References}

1. Royal College of General Practioners - Birmingham Research Unit: Annual prevalence report 2006, Accessed at http://www.rcgp.org.uk/pdf/Annual\% 20prevalence\%20report\%202006.pdf on January 25, 2012.

2. Nuesch E, Dieppe P, Reichenbach $S$, Williams $S$, Iff $S$, Juni P: All cause and disease specific mortality in patients with knee or hip osteoarthritis: population based cohort study. BMJ 2011, 342:d1165.

3. Kivitz AJ, Moskowitz RW, Woods E, Hubbard RC, Verburg KM, Lefkowith JB, Geis GS: Comparative efficacy and safety of celecoxib and naproxen in the treatment of osteoarthritis of the hip. J Int Med Res 2001, 29:467-479.

4. McKenna F, Borenstein D, Wendt H, Wallemark C, Lefkowith JB, Geis GS: Celecoxib versus diclofenac in the management of osteoarthritis of the knee. Scand I Rheumatol 2001, 30:11-18.

5. Zhao SZ, McMillen Jl, Markenson JA, Dedhiya SD, Zhao WW, Osterhaus JT, Yu SS: Evaluation of the functional status aspects of health-related quality of life of patients with osteoarthritis treated with celecoxib. Pharmacotherapy 1999, 19:1269-1278.

6. Rostom A, Moayyedi P, Hunt R: Canadian consensus guidelines on longterm nonsteroidal anti-inflammatory drug therapy and the need for gastroprotection: benefits versus risks. Aliment Pharmacol Ther 2009, 29:481-496.

7. National Institute for Health and Clinical Excellence (NICE): Osteoarthritis: The care and management of osteoarthritis in adults NICE clinical guideline 59 2008.

8. Trelle S, Reichenbach S, Wandel S, Hildebrand P, Tschannen B, Villiger PM, Egger $M$, Juni P: Cardiovascular safety of non-steroidal anti-inflammatory drugs: network meta-analysis. BMJ 2011, 342:c7086.
9. European Medicines Agency: EMEA statement following withdrawal of Vioxx (rofecoxib). EMEA/97949/2004 London 2004.

10. European Medicines Evaluation Agency: EMEA Public statement on the suspension of the marketing authorisation for Bextra (valdecoxib) in the European Union. EMEA/358324/2005 London 2005.

11. European Medicines Agency: European Medicines Agency recommends withdrawal of the marketing authorisations for lumiracoxib containing medicines. EMEA/CHMP/579301/2007 London 2007.

12. Delbecq AL, Vandeven AH: A group process model for problem identification and program planning. J Appl Behav Sci 1971, VIl:466-491.

13. Anderson KM, Wilson PW, Odell PM, Kannel WB: An updated coronary risk profile. A statement for health professionals. Circulation 1991, 83:356-362.

14. Joint British Societies: JBS 2: Joint British Societies' guidelines on prevention of cardiovascular disease in clinical practice. Heart 2005, 91(Suppl 5):v1-52.

15. NHS Clinical Knowledge Service: Nonsteroidal anti-inflammatory drugs (standard or coxibs) - prescribing issues - Management. Who is at increased risk of gastrointestinal adverse effects from oral NSAIDs? 2008, Accessed at http://www.cks.nhs.uk/nsaids_prescribing_issues/management/ detailed_answers/gastrointestinal_adverse_effects/people_at_increased_risk on January 25, 2012.

16. McGettigan P, Henry D: Cardiovascular risk and inhibition of cyclooxygenase: a systematic review of the observational studies of selective and nonselective inhibitors of cyclooxygenase 2. JAMA 2006, 296:1633-1644.

17. Henry D, Lim LL, Garcia Rodriguez LA, Perez Gutthann S, Carson JL, Griffin M, Savage R, Logan R, Moride Y, Hawkey C, et al: Variability in risk of gastrointestinal complications with individual non-steroidal antiinflammatory drugs: results of a collaborative meta-analysis. BMJ 1996, 312:1563-1566.

18. Gladding PA, Webster MW, Farrell HB, Zeng IS, Park R, Ruijne N: The antiplatelet effect of six non-steroidal anti-inflammatory drugs and their pharmacodynamic interaction with aspirin in healthy volunteers. Am J Cardiol 2008, 101:1060-1063.

19. Ballantyne JC, Shin NS: Efficacy of opioids for chronic pain: a review of the evidence. Clin J Pain 2008, 24:469-478.

20. Dunn KM, Hay EM: Opioids for chronic musculoskeletal pain. BMJ 2010, 341:c3533.

21. Solomon DH, Rassen JA, Glynn RJ, Lee J, Levin R, Schneeweiss S: The comparative safety of analgesics in older adults with arthritis. Arch Intern Med 2010, 170:1968-1976.

22. Hippisley-Cox J, Coupland C: Risk of myocardial infarction in patients taking cyclo-oxygenase-2 inhibitors or conventional non-steroidal antiinflammatory drugs: population based nested case-control analysis. BMJ 2005, 330:1366.

23. Gislason GH, Rasmussen JN, Abildstrom SZ, Schramm TK, Hansen ML, Fosbol EL, Sorensen R, Folke F, Buch P, Gadsboll N, et al: Increased mortality and cardiovascular morbidity associated with use of nonsteroidal anti-inflammatory drugs in chronic heart failure. Arch Intern Med 2009, 169:141-149.

24. Schjerning Olsen AM, Fosbol EL, Lindhardsen J, Folke F, Charlot M, Selmer C, Lamberts M, Bjerring Olesen J, Kober L, Hansen PR, et al: Duration of treatment with nonsteroidal anti-inflammatory drugs and impact on risk of death and recurrent myocardial infarction in patients with prior myocardial infarction: a nationwide cohort study. Circulation 2011, 123:2226-2235.

25. Lanas A, Bajador E, Serrano P, Fuentes J, Carreno S, Guardia J, Sanz M, Montoro M, Sainz R: Nitrovasodilators, low-dose aspirin, other nonsteroidal antiinflammatory drugs, and the risk of upper gastrointestinal bleeding. New Engl J Med 2000, 343:834-839.

26. Chan FK, Hung LC, Suen BY, Wu JC, Lee KC, Leung VK, Hui AJ, To KF, Leung WK, Wong WW, et al: Celecoxib versus diclofenac and omeprazole in reducing the risk of recurrent ulcer bleeding in patients with arthritis. New Engl J Med 2002, 347:2104-2110.

27. Lai KC, Chu KM, Hui WM, Wong BC, Hu WH, Wong WM, Chan AO, Wong J, Lam SK: Celecoxib compared with lansoprazole and naproxen to prevent gastrointestinal ulcer complications. Am J Med 2005, 118:1271-1278.

28. Chan FK, Lanas A, Scheiman J, Berger MF, Nguyen H, Goldstein JL: Celecoxib versus omeprazole and diclofenac in patients with osteoarthritis and rheumatoid arthritis (CONDOR): a randomised trial. Lancet 2010, 376:173-179. 
29. Becker MC, Wang TH, Wisniewski L, Wolski K, Libby P, Luscher TF, Borer JS, Mascette AM, Husni ME, Solomon DH, et al: Rationale, design, and governance of Prospective Randomized Evaluation of Celecoxib Integrated Safety versus Ibuprofen Or Naproxen (PRECISION), a cardiovascular end point trial of nonsteroidal antiinflammatory agents in patients with arthritis. Am Heart J 2009, 157:606-612.

30. Pfizer and The Cleveland Clinic: Prospective Randomized Evaluation Of Celecoxib Integrated Safety Vs Ibuprofen Or Naproxen (PRECISION). Clinicaltrials.gov [internet]. National Library of Medicine (US). 2006- [cited June 2 2011] NLM Identifier NCT00346216, Available from: http:/www.clinicaltrials. gov/ct2/show/study/NCT00346216?term=PRECISION+celecoxib\&rank=1 accessed on January 25, 2012.

31. Watkins C, Harvey I, Langley C, Gray S, Faulkner A: General practitioners' use of guidelines in the consultation and their attitudes to them. $\mathrm{Br} J$ Gen Pract 1999, 49:11-15.

32. Woolf SH, Grol R, Hutchinson A, Eccles M, Grimshaw J: Clinical guidelines: potential benefits, limitations, and harms of clinical guidelines. BMJ 1999, 318:527-530.

33. Scheiman JM, Hindley CE: Strategies to optimize treatment with NSAIDs in patients at risk for gastrointestinal and cardiovascular adverse events. Clin Ther 2010, 32:667-677.

34. Antman EM, Bennett JS, Daugherty A, Furberg C, Roberts H, Taubert KA: Use of nonsteroidal antiinflammatory drugs: an update for clinicians: a scientific statement from the American Heart Association. Circulation 2007, 115:1634-1642

Pre-publication history

The pre-publication history for this paper can be accessed here: http://www.biomedcentral.com/1471-2296/13/23/prepub

doi:10.1186/1471-2296-13-23

Cite this article as: Adebajo: Non-steroidal anti-inflammatory drugs for the treatment of pain and immobility-associated osteoarthritis: consensus guidance for primary care. BMC Family Practice 2012 13:23.

\section{Submit your next manuscript to BioMed Central and take full advantage of:}

- Convenient online submission

- Thorough peer review

- No space constraints or color figure charges

- Immediate publication on acceptance

- Inclusion in PubMed, CAS, Scopus and Google Scholar

- Research which is freely available for redistribution

Submit your manuscript at www.biomedcentral.com/submit
Biomed Central 